\title{
Multiparameter spatio-thermochemical probing of flame-wall interactions advanced with coherent Raman imaging
}

\author{
Alexis Bohlin ${ }^{1}$, Christopher Jainski ${ }^{2}$, Brian D. Patterson ${ }^{1}$, Andreas Dreizler ${ }^{2}$, and Christopher J. Kliewer ${ }^{1 *}$ \\ ${ }^{1}$ Combustion Research Facility, Sandia National Laboratories, Livermore, CA 94551, USA \\ ${ }^{2}$ Institute of Reactive Flows and Diagnostics (RSM), 64287 Darmstadt, Germany
}

Christopher J. Kliewer* (Corresponding Author*), cjkliew@sandia.gov, (tel) 00 1-925-294-4973 (fax) 00 1-925-294-2276

Name of Colloquium: Diagnostics (to be reviewed under Coll 15)

Total Length of Paper Using Method 1:

Total Word Equivalent $=\mathbf{5 6 9 5}$

Main Body Text + Figure Captions $=3230+381=3601$

References $=542$

Figures $=506+574+111+146+175=1512$

Equations $=30$

Color Reproduction: Agree to pay color reproduction charges

Submitted for presentation at the

$36^{\text {th }}$ International Symposium on Combustion

Seoul, Korea, July 31-August 5, 2016

1

(C) 2016. This manuscript version is made available under the Elsevier user license http://www.elsevier.com/open-access/userlicense/1.0/ 


\begin{abstract}
Ultrabroadband coherent anti-Stokes Raman spectroscopy (CARS) has been developed for one-dimensional imaging of temperature and major species distributions simultaneously in the near-wall region of a methane/air flame supported on a side-wall-quenching (SWQ) burner. Automatic temporal and spatial overlap of the $\sim 7$ femtosecond pump and Stokes pulses is achieved utilizing a two-beam CARS phase-matching scheme, and the crossed $\sim 75$ picosecond probe beam provides excellent spatial sectioning of the probed location. Concurrent detection of $\mathrm{N}_{2}, \mathrm{O}_{2}, \mathrm{H}_{2}, \mathrm{CO}, \mathrm{CO}_{2}$, and $\mathrm{CH}_{4}$ is demonstrated while high-fidelity flame thermometry is assessed from the $\mathrm{N}_{2}$ pure rotational S-branch in a one-dimensional-CARS imaging configuration. A methane/air premixed flame at lean, stoichiometric, and rich conditions $(\Phi=0.83,1.0$, and 1.2$)$ and Reynolds number $=5,000$ is probed as it quenches against a cooled steel side-wall parallel to the flow providing a persistent flame-wall interaction. An imaging resolution of better than $40 \mu \mathrm{m}$ is achieved across the field-of-view, thus allowing thermochemical states (temperature and major species) of the thermal boundary layer to be resolved to within $\sim 30 \mu \mathrm{m}$ of the interface.
\end{abstract}

Keywords: Time-resolved spectroscopy; CARS; coherent imaging; thermochemical states; flame-wall interactions, combustion diagnostics

\title{
1. Introduction
}

In practical combustion-based devices, such as internal combustion (IC) engines and gas turbines for power generation, there exists significant interaction between gas-phase combustion and metal surfaces. The nature of this heterogeneous interaction is complex, involving both physical and chemical effects. The metal surface is generally much cooler than impinging flames, and significant heat flux to the metal wall ensues during the flamewall interaction (FWI). This heat loss from the gas-phase dramatically slows chemical reaction rates and leads to incomplete combustion. In addition, the structure of the bulk gaseous turbulent flow is necessarily disrupted at a metal wall, creating a feedback loop into the turbulence-dependent flame characteristics such as the local flame velocity. Metal surfaces can additionally increase radical-radical recombination rates. Thus, both the physicaland the chemical processes occurring at metal walls induce local quenching of combustion. The consequences of such FWI have both economic and environmental impacts. Device ageing and failure is often related to local 
dynamical heat load, while incomplete combustion leads to increased emission of pollutants such as unburned hydrocarbons and $\mathrm{CO}$.

In order to make the study of FWI tractable both experimentally and computationally, laboratory burner geometries have been developed to allow for well-controlled boundary conditions, optimal optical access, and isolation of parameter dependencies. Both head-on-quenching (HOQ) and side-wall quenching (SWQ) burners have been utilized. In the HOQ arrangement, a flame propagates to and impinges perpendicularly against a metal wall, where it is quenched. HOQ is therefore a transient quenching process. In the SWQ burner design, the bulk flow of the combusting gases travels parallel to the wall. If flame stabilization is established in the convective flow, this arrangement allows for a persistent and statistically stationary FWI to be studied in both laminar and turbulent conditions. Clearly, in reality FWI for practical combustion devices will exhibit both HOQ and SWQ behaviors.

In practical combustion systems, power densities and surface-to-volume ratios are ever-increasing in pursuit of enhanced efficiency with reduced harmful emissions, increasing the importance of FWI [1]. For this reason, a much improved understanding of FWI is needed. Thermochemical states during FWI have been investigated for a long time by one-dimensional direct numerical simulation (DNS) including detailed chemistry and transport models for the case of head-on quenching. These works are summarized in a recent review [2]. DNS including detailed chemistry and transport has been extended only recently by Gruber et al. [3] to three spatial dimensions. To date, 3D-DNS has been restricted to hydrogen combustion to simplify the chemistry thereby limiting computational costs. Consequently, pollutant generation such as CO-formation has not yet been addressed at all in DNS, although it is one of the clear motivating factors for increased understanding of FWI. Experimentally, Mann et al. [4] have recently performed simultaneous temperature-CO-measurements in a HOQ burner as a first step to investigate finite-rate chemistry effects near walls. We have recently reported on near-wall temperature measurements utilizing a two-beam femtosecond / picosecond 1D-CARS configuration to study transient quenching in a HOQ burner [5]. Access to the full 1D temperature profile in each laser shot allows access to spatially correlated statistics with the elevated accuracy and precision achievable in CARS-based assessment. Head-on quenching of premixed flames, however, is inherently a transient phenomenon: after its initiation the 
flame propagates towards the wall and is quenched. For any statistical evaluation of FWI under turbulent flow conditions this process must be repeated many times. For numerical as well experimental analysis this poses a severe limitation. Investigating thermochemical states in FWI for statistically stationary conditions is thus highly desirable for generating experimental benchmark datasets for comparison to combustion simulation.

One of the most urgent needs in the study of FWI is the development of experimental techniques for multiparameter measurements, focusing on turbulent heat flux and thermochemical states [2]. In the present work, we demonstrate the feasibility of a supercontinuum-based 1D-CARS approach to extend experimental studies of thermochemical states towards SWQ, allowing the mapped temperature distribution across the thermal boundary layer to be correlated with the distribution of all major Raman-active species. Ultrabroadband twobeam 1D-CARS [6] has been recently developed and benchmarked in a canonical sooting flat-flame [7]. The key advantage of the technique is the simultaneous acquisition of high-fidelity thermometry with wideband chemical detection for major species. The advance utilizes the simplified two-beam 1D-CARS phase matching arrangement [8] for robust signal generation and the inherent automatic overlap of the pump and Stokes fields. A prerequisite for this advance was the development of hybrid femtosecond/picosecond-CARS for combustion applications [9-11]. The extreme efficiency of driving the coherence generation with transform limited fs pulses yields extraordinarily high signal levels amenable to developments in CARS imaging [8, 12-14]. The presented measurements have a spatial resolution of $\sim 35 \mu \mathrm{m}$ in the wall-normal direction and data is resolved to within $\sim 30$ $\mu \mathrm{m}$ of the metal surface.

\section{Experimental}

SWQ burner setup: A generic burner setup was designed to experimentally investigate side-wall flame quenching for laminar and turbulent flow conditions. The burner consists of a Morel-shaped quadratic jet nozzle [15] with an exit area of $40 \times 40 \mathrm{~mm}^{2}$. Sharp edges at the nozzle exit minimize recirculation zones. Upstream of the contraction the premixed methane/air mixture enters a settling chamber, before it is homogenized by two sets of fine meshes and an additional honeycomb structure. This central flow is surrounded by an air co-flow operated with an outlet velocity of $75 \%$ of the bulk velocity of the jet. The contoured nozzle provides a top-hat exit velocity profile. A circular ceramic rod $(\varnothing 1 \mathrm{~mm})$ is placed $16 \mathrm{~mm}$ downstream the nozzle exit to stabilize a V-shaped flame. One 4 
branch of the flame interacts with a vertically orientated steel wall. The wall is temperature stabilized by internal water cooling and is slightly curved (radius $=300 \mathrm{~mm}$ ) for optimal optical access. In the flame -wall interaction region SWQ is observed. Methane (purity 99.9\%) and dried air are perfectly mixed before entering the nozzle assembly. Flow rates of both gas components are controlled by electronic mass flow controllers (MKS) and can be varied in a wide range spanning from laminar to turbulent conditions. The mass flow controllers were calibrated with a flow verifier (MKS type GBROR). The nozzle is surrounded by coflowing dry air to minimize mixing with ambient air. In the case without a turbulence grid the flame burns laminar with an almost stationary flame-wall interaction section. Due to the burner geometry Helmholtz resonances at a frequency of $104 \mathrm{~Hz}$ occur. For the present operational conditions this causes vertical fluctuations of the flame tip of $+/-200 \mu \mathrm{m}$ at the wall. All experiments are performed at a Reynolds number of 5,000 (based on the hydraulic diameter of the nozzle exit), and equivalence ratios of $\varphi=0.83,1$, and 1.2 .

CARS Setup: The two-beam 1D-CARS experiments are implemented in two different arrangements, as reported previously [7]. In the first set of experiments, the output of a femtosecond regenerative amplifier (Wyvern 1000, KM Labs) consisting of a 45 femtosecond pulse duration is used directly as the pump/Stokes pulse, with $\sim 3$ $\mathrm{mJ} /$ pulse. The high energy $(\sim 30 \mathrm{~mJ} /$ pulse $)$ picosecond probe pulse at $532 \mathrm{~nm}$ originates from a separate Nd:YAG-based regenerative amplifier. The $100 \mathrm{MHz}$ repetition rate of the oscillators for both the femtosecond and picosecond lasers are phase-locked to an external RF source, allowing for precise electronic timing of the pulses at the experiment with low jitter ( $<1$ picosecond). The $\sim 75$ picosecond probe pulse duration was routinely measured through a CARS cross-correlation performed in argon. The femtosecond $(\mathrm{f}=500 \mathrm{~mm})$ and picosecond $(\mathrm{f}=300 \mathrm{~mm})$ beams are focused with cylindrical optics to the experimental probe volume, forming horizontal sheets perpendicular to the flame side-wall. The 1D-CARS signal generation volume is formed where the two sheets (pump/Stokes and probe) intersect. The horizontal signal sheet is then relay imaged through a turning periscope to the vertical spectrometer slit of an imaging spectrometer (Horiba, iHR550). The signal beam is isolated from the copropagating probe beam with two angle-tuned short pass filters (Semrock). The dispersed signal is detected on a water-cooled CCD (Andor, Newton) with $16 \mu \mathrm{m}$ pixel size. An example of the signal image from this arrangement is given in Figure 1e. 

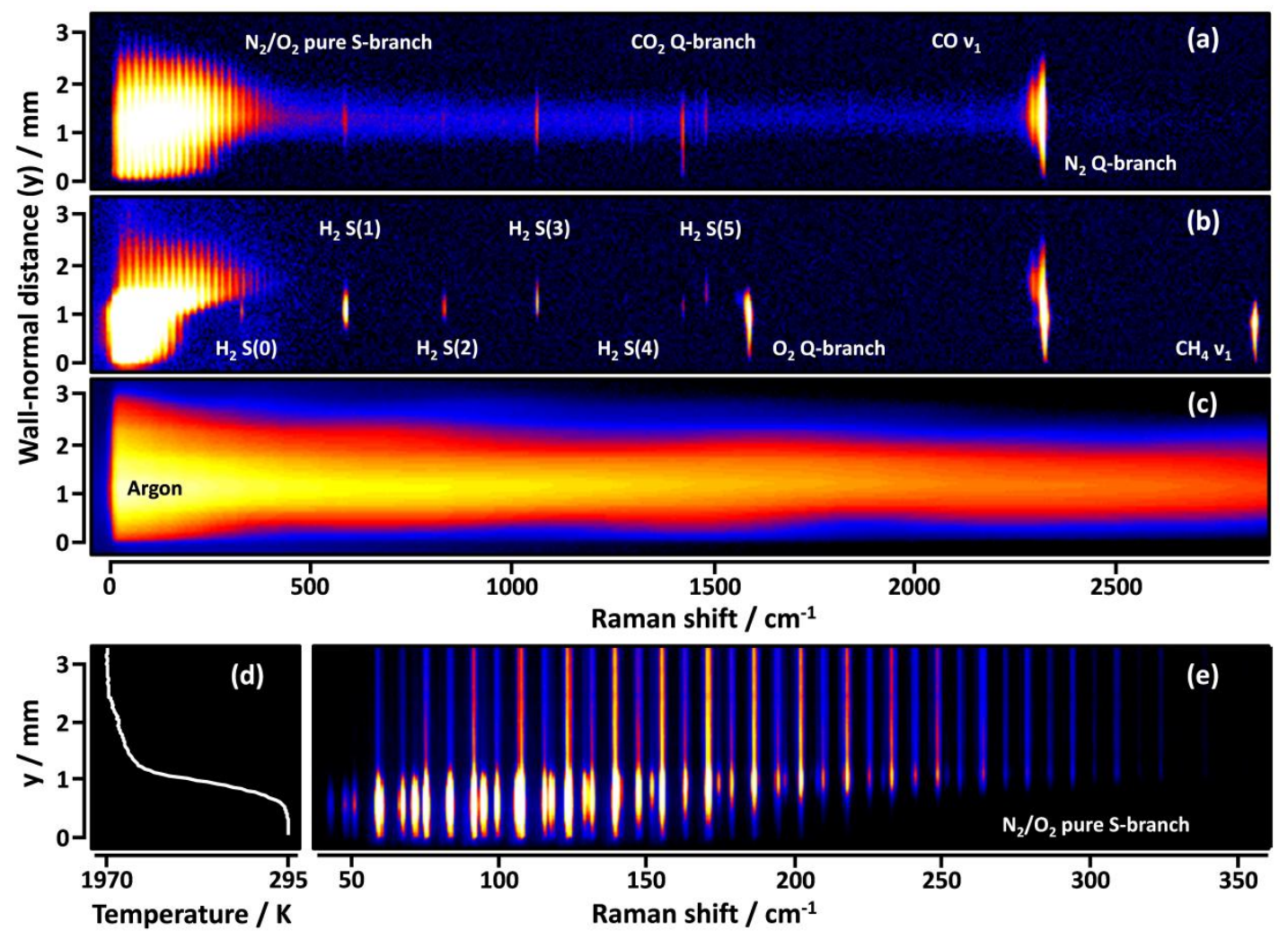

Fig.1. CCD image of the 1D-CARS signal obtained in the operating SWQ burner from 500 averaged laser shots demonstrating the capability for simultaneous imaging of temperature and ultrabroadband species detection. (a) Data taken in the product zone, $\Phi=1.2, \mathrm{HAB}=50 \mathrm{~mm}$, probe delay $=60$ picoseconds. (b) Data taken in the FWI region, $\Phi=1.2, \mathrm{HAB}=39.5 \mathrm{~mm}$, probe delay $=200$ picoseconds. (c) Image taken in a flow of argon to assess the effective excitation profile of the ultrabroadband laser. (d) Evaluated temperature profile from the S-branch $\mathrm{N}_{2}$ CARS data presented in panel (e) at $\Phi=0.83, \mathrm{HAB}=55 \mathrm{~mm}$, probe delay $=200$ picoseconds.

The 1D spatial coordinate of the probe volume is mapped to the vertical axis of the CCD, while the horizontal axis represents the frequency-resolved spectral shift. Individual pure-rotational transitions of $\mathrm{N}_{2}$ and $\mathrm{O}_{2}$ are observed. It was discovered while performing the experiments that even subtle reflections from the wall can have an impact on the evaluation of CARS spectra in the near-wall region. Surprisingly, even in the case where no obvious reflection or diffraction effects were present in the signal image on the $\mathrm{CCD}$, subsequent evaluation of the spectra would at times reveal inaccuracies in the evaluated temperature profile in careful calibration experiments both in room air and in operating flames. To minimize this effect, razor blade edges are inserted into the beam paths of the pump/Stokes and probe laser beams. The razor blade edge plane is relay imaged in a 4-f 
arrangement to the probe volume location where the two laser sheets intersect. When the image plane is aligned optimally, the diffraction from the blade edge converges at the probe-volume beam intersection and the sharp edge on the laser sheets minimizes the effects of wall reflections. We have found this to be a critical experimental consideration for obtaining accurate temperature profiles in near-wall regions. Based on translation of the wallburner assembly and razor blades while monitoring the cutoff of the 1D-CARS image, it was determined that spatial locations to within $\sim 30 \mu \mathrm{m}$ of the surface are assessed with good accuracy. Because of flame luminosity, a chopper wheel was inserted at the entrance slit of the spectrometer to eliminate the majority of background signals.

In the second experimental arrangement, a portion $(\sim 2 \mathrm{~mJ})$ of the output of the femtosecond regenerative amplifier is focused into a hollow-core fiber 1-meter in length with a $310 \mu \mathrm{m}$ fiber core diameter (Kaleidoscope, Femtolasers). The fiber is housed in a pressure vessel of $\sim 600$ Torr Ar. The femtosecond pulse is significantly frequency broadened by self-phase modulation as it propagates through the waveguide. A set of mirrors imparting negative chirp are placed after the fiber, thereby compressing the pulse to ultrashort $<7$ femtoseconds as measured by autocorrelation (Femtometer, Femtolasers). The $\sim 0.8 \mathrm{~mJ}$ ultrashort pump/Stokes beam is then reflected from a pair of two cylindrical concave $\mathrm{f}=400 \mathrm{~mm}$ silver mirrors, mounted with opposite axial focusing, and a last turning periscope consisting of two flat silver mirrors sending the pump/Stokes beam to the crossing. As described previously [16], the introduced astigmatism on the pump/Stokes beam enhances the irradiance across the field of view by compressing the spatial extent of the sheet. The beams are crossed at an angle of $\sim 5^{\circ}$, yielding a negligible phase-mismatch effect and an interaction length of $\sim 400 \mu \mathrm{m}$.

In all experiments, the effective spectral excitation profile of the femtosecond pulses is measured by recording a nonresonant 1D-CARS image in argon with time-overlapped pump/Stokes and probe pulses. Figure 1c displays the ultrabroadband excitation profile obtained with the 7 femtosecond pump/Stokes pulse. The frequency bandwidth of this laser pulse covers the fundamental rotational and vibrational transitions of most molecules, and thus provides for a generic experimental platform for detecting multiple species in a CARS experiment independent of the specific chemistry involved. Figure 1a and 1b demonstrate measurements in the SWQ burner 
taken with the 7 femtosecond excitation source under the rich flame condition $(\Phi=1.2)$. Figure 1a is recorded in the product zone after quenching at a height above the burner nozzle $(\mathrm{HAB})$ of $50 \mathrm{~mm}$. It demonstrates the detection of the spatial distribution of $\mathrm{N}_{2}, \mathrm{H}_{2}, \mathrm{CO}$ and $\mathrm{CO}_{2}$. Figure $1 \mathrm{~b}$ is taken closer to the FWI location at HAB $39.5 \mathrm{~mm}$, and demonstrates the detection of $\mathrm{N}_{2}, \mathrm{O}_{2}, \mathrm{H}_{2}$ and $\mathrm{CH}_{4}$.

Flame Measurements: The methane/air flame is operated at lean, stoichiometric, and rich conditions $(\Phi=0.83$, 1.0, and 1.2). 1D-CARS temperature profiles have been acquired at different heights above the burner nozzle (HAB), with $500 \mu \mathrm{m}$ height separation between each profile obtaining the dynamics of the flame quenching in sequence. At each HAB, 500 single-laser shot CARS images with corresponding background have been recorded.

\section{Theoretical Considerations}

The time-domain rotational CARS code used for generating the spectral library is similar to that presented in earlier works $[10,17,18]$. The probe delay in these temperature experiments was generally set to 200 picoseconds. This ensured the full suppression of the nonresonant background as well as a balanced detection of signals imaged simultaneously from both cold and hot zones of the reacting flow [8]. The nonresonant susceptibility has been omitted from the calculation of the CARS response, as it is negligible for such long probe delays in femtosecond/picosecond CARS. For these experiments, the modified exponential gap law [19] has been used for determining the $\mathrm{N}_{2}$ linewidths used in the calculation for temperature over $1400 \mathrm{~K}$, which is based upon $\mathrm{N}_{2}$ self-broadening linewidths and the parameter set used can be found in [20]. Below $1400 \mathrm{~K}$, interpolated linewidths based on those measured in time-domain CARS experiments [21] were directly used in simulating the library of spectra. Herman-Wallis factors have been employed to address the breakdown of the rigid-rotor approximation [22]. The experimental spectra are corrected for the excitation efficiency and any transmission characteristics of the signal detection path by dividing the measured spectra by a spectrum recorded in pure argon. Because of the large number of CARS spectra to be fitted, a non-linear interpolating method based on a spectral library is required, determining the temperature from a least-square error minimization fitting procedure, 
commonly employed in nanosecond CARS [23]. The contour of the integrated line-intensities from each of the library spectra are normalized and compared to the distribution of the measured spectrum in the fitting routine.

\section{Results and Discussion}

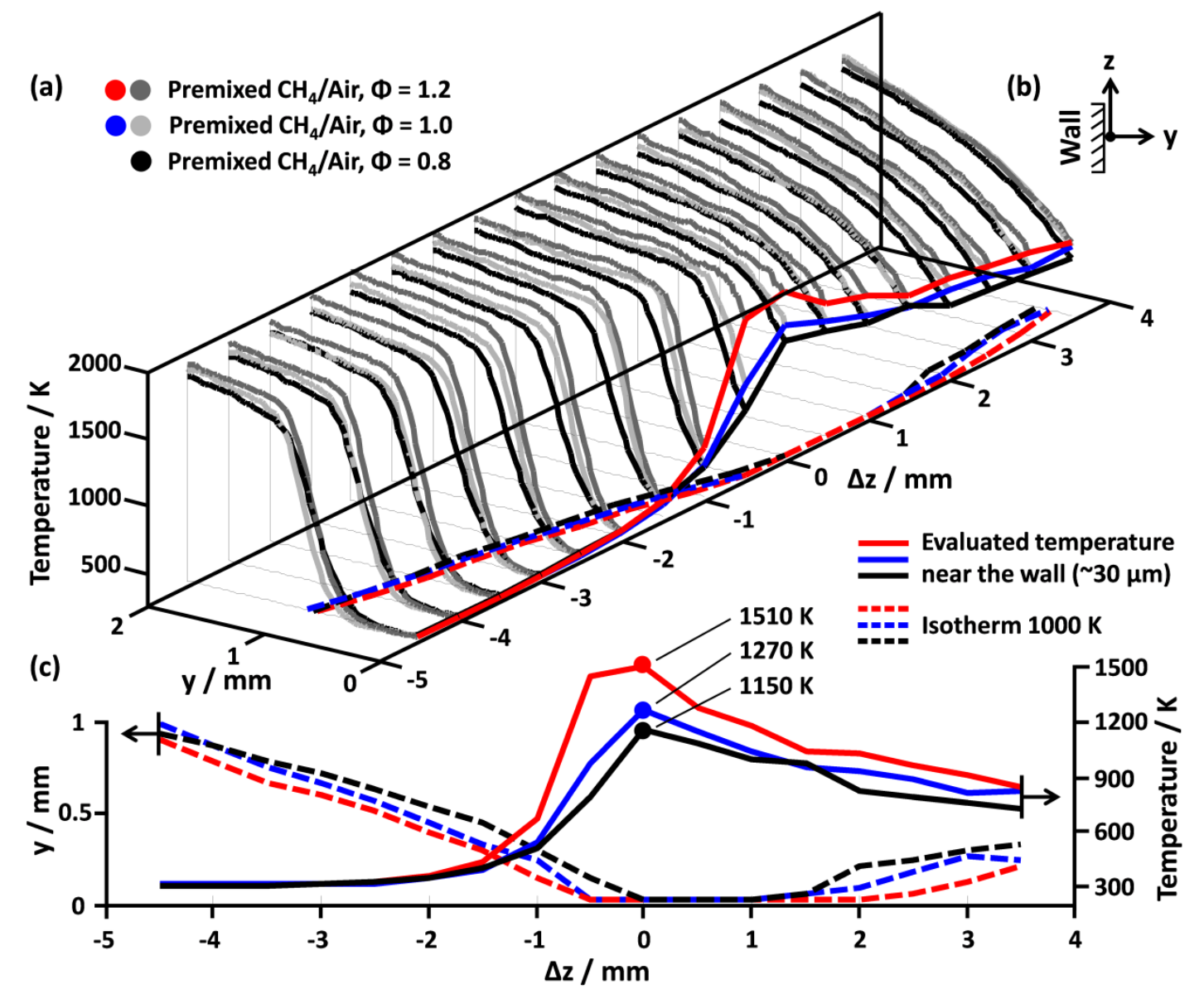

Fig. 2. (a) Evaluated $1 \mathrm{D}$ mean temperature profiles at different $\mathrm{HAB}$ for $\Phi=0.83,1.0$, and 1.2. As described by the inset (b), the y-axis corresponds to distance in the wall normal direction, and the z-axis corresponds to the spatial coordinate over the burner nozzle. The values for $\mathrm{z}$ have been shifted such that position 0 corresponds to the point where the flame impinges on the side-wall. This offset corresponds to $60.5,48.5$, and $46.0 \mathrm{~mm} \mathrm{HAB}$ for $\Phi=0.83,1.0$, and 1.2, respectively. Both the evaluated temperature near the wall $(\sim 30 \mu \mathrm{m})$ and position of the $1000 \mathrm{~K}$ isotherm are shown and depicted more clearly in panel (c).

The evaluation of the two-beam 1D-CARS data for near-wall thermometry in the SWQ burner is presented in Figure 2. As visualized in the inset, Figure $2 b$, the y-axis corresponds to the wall-normal distance (mm) while the z-axis corresponds to the HAB. However, the position 0-mm on the z-axis has been shifted to the location where 
the flame impinges against the metal wall. This position occurs at HAB $60.5,48.5$, and $46.0 \mathrm{~mm}$ for $\Phi=0.83,1.0$, and 1.2, respectively. This flame-based coordinate system thus allows comparison of the thermal boundary layer structure for the three reaction mixtures. In Figure $2 \mathrm{a}$, at negative $\mathrm{z}$ values, the dynamics of the flame as it approaches and quenches at the wall are observed. At positive $\mathrm{z}$ values, one can observe how the wall-normal temperature profiles change with reduced thermal gradients (with maximum gradient occurring at the wall) after the quenching interaction. The maximum flame temperatures, extracted at a distance of $\sim 4 \mathrm{~mm}$ away from the wall, have been evaluated to be $1970 \mathrm{~K}, 2190 \mathrm{~K}$, and $2150 \mathrm{~K}$ for $\Phi=0.83,1.0$, and 1.2 , respectively, which is $\sim 1 \%$ below the adiabatic flame temperature. Figure $2 \mathrm{c}$ presents the near-wall temperatures evaluated as a function of $\mathrm{HAB}$ for the three stoichiometric conditions, as well as following the progression of the $1000 \mathrm{~K}$ isotherm for the three cases. As can be seen the highest near-wall temperatures are observed for the richest case. In numerical simulations of a HOQ burner, Popp and Baum [24] concluded that at elevated wall temperatures ( > $400 \mathrm{~K}$ ) surface reactions should no longer be ignored even on 'inert' walls in simulating the FWI. The high near-wall temperatures measured here, in combination with the prolonged surface interaction of the SWQ burner arrangement, motivates future work to evaluate the wall temperature and role of surface mediated reactions on the FWI.

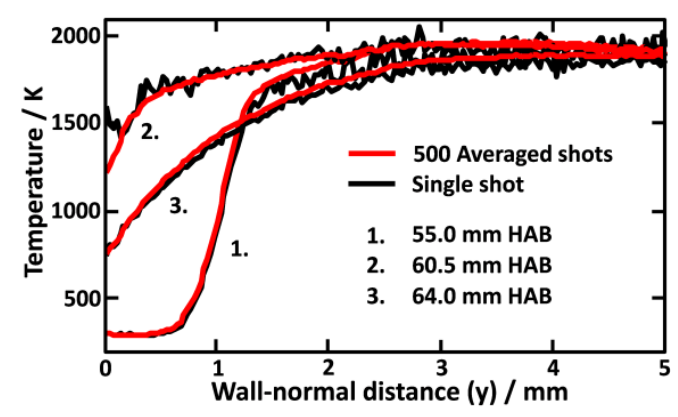

Fig. 3. Comparison between the evaluated 1D-CARS temperature profiles obtained in the averaged case (500 laser shots) and in a single laser shot assessment. $\Phi=0.83$. The three $\operatorname{HAB}(55,60.5,64 \mathrm{~mm})$ correspond to spatial locations before quenching, during quenching, and post-quenching respectively.

The capability for single-shot $1 \mathrm{D}$ thermometry measurements is presented for the lean flame condition in Figure 3. The red lines present the results of 500 averaged shots at three different HAB corresponding to positions 10 
before, during, and after quenching (line 1,2, and 3, respectively). Representative single-laser-shot assessments are included in black for the corresponding HAB. The precision in the single-shot temperature assessment across the field-of-view from the current femtosecond/picosecond CARS setup is $~ 2-3 \%$ operating in flames, as shown previously [7].

In addition to high-fidelity thermometry throughout the thermal boundary layer, the current ultrabroadband CARS imaging technique additionally provides the capability for mapping the distribution of main chemical species ratios. As shown in Figure 1, signals from $\mathrm{N}_{2}, \mathrm{O}_{2}, \mathrm{CH}_{4}, \mathrm{H}_{2}, \mathrm{CO}_{2}$, and $\mathrm{CO}$ are observable in the currently studied conditions of the SWQ burner. This new capability begins to address one of the most urgent needs for model validation data in FWI studies, the determination of thermo-chemical state conditioned statistics. Once the species resonances are corrected for the excitation efficiency, the species mole fraction ratios may be evaluated according to Equation 1:

$$
\frac{X_{i}}{N_{2}}=\sqrt{\frac{S_{X_{i}}}{S_{N_{2}}}} \times \frac{\sigma_{N_{2}}}{\sigma_{X_{i}}} \times e^{-t\left(1 / \tau_{N_{2}}-1 / \tau_{X_{i}}\right)}
$$

where $X_{i} / N_{2}$ is the molar ratio of the $i$-th chemical species relative to $\mathrm{N}_{2}, S_{X_{i}} / S_{N_{2}}$ is the ratio of the integrated CARS signal of species $i$ compared to $\mathrm{N}_{2}, \sigma_{X_{i}}$ is the Raman cross section for species $i, t$ is the probe pulse delay time (100 ps for the concentration measurements), and $\tau_{X_{i}}$ is the dephasing constant for species $i$. The species mole fraction ratio (relative to inert $\mathrm{N}_{2}$ ) is commonly employed instead of the absolute species mole fractions, since the difficulty of detecting $\mathrm{H}_{2} \mathrm{O}$ in CARS multiplexed techniques [25-27]. The relative Raman cross sections [28] and the dephasing constants for each species-specific transition must be obtained from the literature or measured. It has been shown that the collisional coherence dephasing constant may be measured in-situ in flames with a probe delay scan technique which negates the need for a linewidth model [29]. Here, these probe delay scans were performed measuring spatially dependent total collisional dephasing rates for each detected species in the wall-normal direction. With the dephasing constant known, the integrated signal intensities can be corrected for the probe delay. 


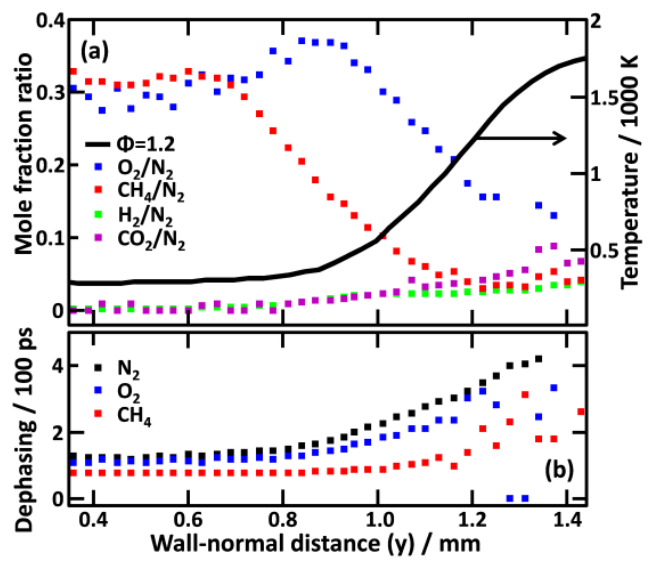

Fig. 4. (a) Evaluation of the mole fraction ratios for $\mathrm{O}_{2}, \mathrm{CH}_{4}, \mathrm{H}_{2}$, and $\mathrm{CO}_{2}$ all relative to $\mathrm{N}_{2}$ in the SWQ burner at $\Phi=1.2$. The corresponding temperature profile evaluated from the spectral fitting of the $\mathrm{N}_{2} \mathrm{~S}$-branch is also shown. (b) In-situ measurement of the total collisional dephasing constant across a $\sim 1 \mathrm{~mm}$ line segment is presented for several species.

In Figure 4a, the mole fraction ratios for $\mathrm{O}_{2} / \mathrm{N}_{2}, \mathrm{CH}_{4} / \mathrm{N}_{2}, \mathrm{H}_{2} / \mathrm{N}_{2}$, and $\mathrm{CO}_{2} / \mathrm{N}_{2}$ are presented for the rich SWQ case at $\mathrm{HAB} 39.5 \mathrm{~mm}$. Additionally, Figure 5 presents joint profiles for the spatial evolution of the thermochemical state evaluated at the $1000 \mathrm{~K}$ isotherm for $\mathrm{O}_{2}, \mathrm{H}_{2}, \mathrm{CH}_{4}$, and $\mathrm{CO}_{2}$ during $\mathrm{FWI}$, as an example of the type of conditional statistics possible with the new technique. Further work in our laboratory is underway to refine the mole fraction ratio calculation, for instance, by measuring the temperature dependent relative Raman cross sections, as significant spread exists for the values reported in the literature.

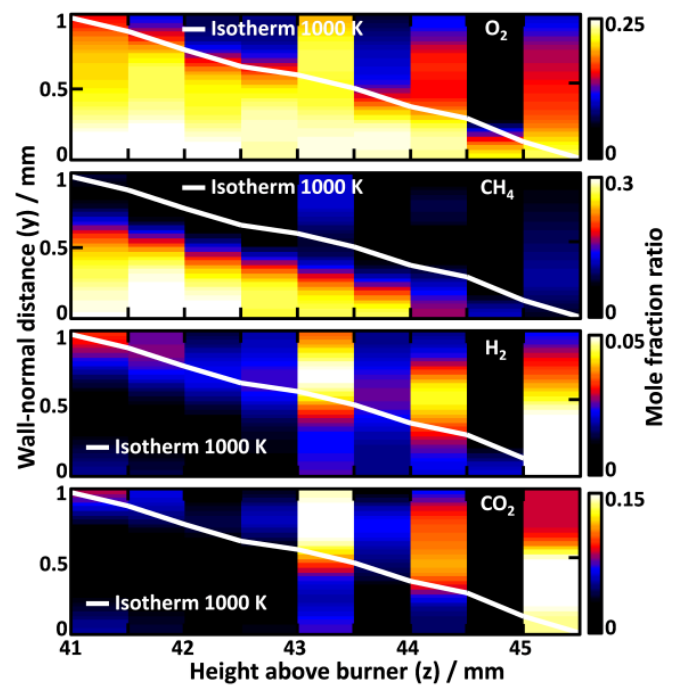

Fig. 5. Direct measurement of mean thermochemical states during FWI. The evaluated mole fraction ratio distributions in the thermal boundary layer are presented at the $1000 \mathrm{~K}$ isotherm for $\mathrm{O}_{2}, \mathrm{CH}_{4}, \mathrm{H}_{2}$, and $\mathrm{CO}_{2}$. 


\section{Summary}

Two-beam femtosecond/picosecond 1D-CARS was utilized to provide high-fidelity thermometry across the thermal boundary layer of SWQ methane/air flames supported on a generic burner designed to create a statistically stationary FWI. Robust methods for removing the artifacts caused by beam reflections from the surface have been successfully developed. In addition, implementing an ultrashort femtosecond driving pulse has enabled the simultaneous measurement of the major flame species jointly with the assessed thermometry, demonstrated for detection of $\mathrm{N}_{2}, \mathrm{O}_{2}, \mathrm{H}_{2}, \mathrm{CO}, \mathrm{CO}_{2}$, and $\mathrm{CH}_{4}$. This development provides unique access to conditional statistics on the thermo-chemical state during FWI processes.

\section{Acknowledgements}

This material is based upon work supported by an award from the United States Department of Energy's Office of Science Early Career Research Program. Development of the femtosecond/picosecond CARS system was supported by Office of Chemical Sciences, Biosciences, and Geosciences, Office of Basic Energy Sciences, of the

U. S. Department of Energy. Sandia is a multiprogram laboratory operated by Sandia Corporation, a Lockheed Martin Company, for the U.S. Department of Energy's National Nuclear Security Administration under contract DE-AC04-94AL85000. C. Jainski and A. Dreizler gratefully acknowledge financial support by Deutsche Forschungsgemeinschaft through SFB/TRR 150. In addition A. Dreizler is thankful for support through the Gottfried Wilhalm Leibniz-program.

\section{References}

[1] A.C. Alkidas, Prog. Energy Combust. Sci., 25 (1999) 253-273.

[2] A. Dreizler, B. Bohm, Proc. Combust. Inst., 35 (2015) 37-64.

[3] A. Gruber, R. Sankaran, E.R. Hawkes, J.H. Chen, J. Fluid Mech., 658 (2010) 5-32.

[4] M. Mann, C. Jainski, M. Euler, B. Bohm, A. Dreizler, Combust. Flame, 161 (2014) 2371-2386.

[5] A. Bohlin, M. Mann, B.D. Patterson, A. Dreizler, C.J. Kliewer, Proc. Combust. Inst., 35 (2015) 3723-3730.

[6] A. Bohlin, C.J. Kliewer, Appl. Phys. Lett., 104 (2014) 5.

[7] A. Bohlin, C.J. Kliewer, J. Phys. Chem. Lett., 6 (2015) 643-649.

[8] A. Bohlin, B.D. Patterson, C.J. Kliewer, J. Chem. Phys., 138 (2013) 081102.

[9] S.R. Engel, J.D. Miller, C.E. Dedic, T. Seeger, A. Leipertz, T.R. Meyer, J. Raman Spectrosc., 44 (2013) 13361343.

[10] J.D. Miller, S. Roy, M.N. Slipchenko, J.R. Gord, T.R. Meyer, Opt. Express, 19 (2011) 15627-15640. 
[11] B.D. Prince, A. Chakraborty, B.M. Prince, H.U. Stauffer, J. Chem. Phys., 125 (2006).

[12] C.J. Kliewer, Y. Gao, T. Seeger, B.D. Patterson, R.L. Farrow, T.B. Settersten, Appl. Opt., 50 (2011) 1770-1778.

[13] W.D. Kulatilaka, H.U. Stauffer, J.R. Gord, S. Roy, Opt. Lett., 36 (2011) 4182-4184.

[14] A. Bohlin, C.J. Kliewer, J. Chem. Phys., 138 (2013) 221101.

[15] T. Morel, J. Fluids Eng.-Trans. ASME, 97 (1975) 225-233.

[16] A. Bohlin, C.J. Kliewer, Appl. Phys. Lett., 105 (2014) 5.

[17] S.P. Kearney, D.J. Scoglietti, C.J. Kliewer, Opt. Express, 21 (2013) 12327-12339.

[18] S. Roy, W.D. Kulatilaka, D.R. Richardson, R.P. Lucht, J.R. Gord, Opt. Lett., 34 (2009) 3857-3859.

[19] M.L. Koszykowski, L.A. Rahn, R.E. Palmer, M.E. Coltrin, J. Phys. Chem., 91 (1987) 41-46.

[20] L.A. Rahn, R.E. Palmer, J. Opt. Soc. Am. B-Opt. Phys., 3 (1986) 1164-1169.

[21] C.J. Kliewer, A. Bohlin, E. Nordstrom, B.D. Patterson, P.E. Bengtsson, T.B. Settersten, Appl. Phys. B-Lasers Opt., 108 (2012) 419-426.

[22] A. Bohlin, P.E. Bengtsson, M. Marrocco, J. Raman Spectrosc., 42 (2011) 1843-1847.

[23] A. Bohlin, E. Nordström, H. Carlsson, X.-S. Bai, P.-E. Bengtsson, Proc. Combust. Inst., 34 (2013) 3629-3636.

[24] P. Popp, M. Baum, Combust. Flame, 108 (1997) 327-348.

[25] F. Vestin, P.E. Bengtsson, Proc. Combust. Inst., 32 (2009) 847-854.

[26] S.A. Tedder, J.L. Wheeler, A.D. Cutler, P.M. Danehy, Appl. Opt., 49 (2010) 1305-1313.

[27] C.E. Dedic, J.D. Miller, T.R. Meyer, Opt. Lett., 39 (2014) 6608-6611.

[28] D.G. Fouche, R.K. Chang, Appl. Phys. Lett., 20 (1972) 256-\&.

[29] Y. Gao, A. Bohlin, T. Seeger, P.E. Bengtsson, C.J. Kliewer, Proc. Combust. Inst., 34 (2013) 3637-3644. 


\section{Figure Captions}

Figure 1. CCD image of the 1D-CARS signal obtained in the operating SWQ burner from 500 averaged laser shots demonstrating the capability for simultaneous imaging of temperature and ultrabroadband species detection. (a) Data taken in the product zone, $\Phi=1.2, \mathrm{HAB}=50 \mathrm{~mm}$, probe delay $=60 \mathrm{ps}$. (b) Data taken in the FWI region, $\Phi=1.2, \mathrm{HAB}=39.5 \mathrm{~mm}$, probe delay $=200 \mathrm{ps}$. (c) Image taken in a flow of argon to assess the effective excitation

profile of the ultrabroadband laser. $\quad$ (d) Evaluated temperature profile from the S-branch $\mathrm{N}_{2}$ CARS data presented in panel (e) at $\Phi=0.83, \mathrm{HAB}=55 \mathrm{~mm}$, probe delay $=200 \mathrm{ps}$.

Figure 2. Fig. 2. (a) Evaluated $1 \mathrm{D}$ mean temperature profiles at different $\mathrm{HAB}$ for $\Phi=0.83,1.0$, and 1.2. As described by the inset (b), the y-axis corresponds to distance in the wall normal direction, and the $\mathrm{z}$-axis corresponds to the spatial coordinate over the burner nozzle. The values for $\mathrm{z}$ have been shifted such that position 0 corresponds to the point where the flame impinges on the side-wall. This offset corresponds to $60.5,48.5$, and $46.0 \mathrm{~mm}$ HAB for $\Phi=0.83,1.0$, and 1.2 , respectively. Both the evaluated temperature near the wall $(\sim 40 \mu \mathrm{m})$ and position of the $1000 \mathrm{~K}$ isotherm are shown and depicted more clearly in panel (c).

Figure 3. Comparison between the evaluated 1D-CARS temperature profiles obtained in the averaged case (500 laser shots) and in a single laser shot assessment. $\Phi=0.83$. The three $\operatorname{HAB}(55,60.5,64 \mathrm{~mm})$ correspond to spatial locations before quenching, during quenching, and post-quenching respectively.

Figure 4. (a) Evaluation of the mole fraction ratios for $\mathrm{O}_{2}, \mathrm{CH}_{4}, \mathrm{H}_{2}$, and $\mathrm{CO}_{2}$ all relative to $\mathrm{N}_{2}$ in the SWQ burner at $\Phi=1.2$. The corresponding temperature profile evaluated from the spectral fitting of the $\mathrm{N}_{2} \mathrm{~S}$-branch is also shown. (b) In-situ measurement of the total collisional dephasing constant across a $\sim 1 \mathrm{~mm}$ line segment is presented for several species. (c) The spatial evolution of the thermochemical state is visualized from the evaluated data with a $1000 \mathrm{~K}$ isotherm, and the position of the inflection point in the $\mathrm{O}_{2} / \mathrm{N}_{2}$ ratio as well as the $\mathrm{CH}_{4} / \mathrm{N}_{2}$ ratio. 
Figure 5. Direct measurement of mean thermochemical states during FWI. The evaluated mole fraction ratio distributions in the thermal boundary layer are presented at the $1000 \mathrm{~K}$ isotherm for $\mathrm{O}_{2}, \mathrm{CH}_{4}, \mathrm{H}_{2}$, and $\mathrm{CO}_{2}$. 